\title{
Neurovascular Behçet's Disease Presenting With Cavernous Sinus Thrombosis: A Rare Case of Assumed Tolosa-Hunt-Like Syndrome
}

\author{
Chong Hyuk CHUNG ${ }^{\circledR}$, Myeung Su LEE $[$, Changhoon LEE $(\mathbb{D}$ \\ Department of Internal Medicine, Wonkwang University Hospital, Iksan, South Korea
}

\begin{abstract}
Behçet's disease (BD) is a rare, chronic, auto-inflammatory disorder of unknown origin. Mucocutaneous lesions and pan-uveitis constitute the hallmark of BD. Additionally, vessels of all sizes and types are affected. In this article, we report a 53-year-old female patient diagnosed with neurovascular BD with diplopia, ipsilateral headache and ophthalmoplegia associated with cavernous sinus thrombosis, leading to Tolosa-Hunt-like syndrome. To our knowledge, this is the first report of a patient with complicated cavernous sinus thrombosis in BD.

Keywords: Cavernous sinus thrombosis, neurovascular Behçet's disease, Tolosa-Hunt-like syndrome.
\end{abstract}

Behçet's disease (BD) is characterized by recurrent orogenital ulcers, uveitis and various neutrophilic dermatoses. The histopathological feature of $\mathrm{BD}$ includes vasculitis involving veins and arteries of distinct sizes. ${ }^{1}$ Approximately 5 to $6 \%$ of all BD cases manifest various neurological symptoms. The prevalence of cerebral venous sinus thrombosis (CVST) in BD is $8 \%$, which corresponds to $18 \%$ of neurovascular BD (neuro-BD). Sites frequently involved include the superior sagittal (64\%) and the transverse sinus (61\%). ${ }^{2}$ However, the cavernous sinus involvement has been very rarely reported.

Tolosa-Hunt syndrome (THS) is a rare corticosteroid-responsive etiology associated with painful ophthalmoplegia characterized by unilateral orbital pain with paresis of one or more of the third, fourth, and sixth cranial nerves, commonly leading to diplopia. It is an idiopathic granulomatous inflammation involving the cavernous sinus/superior orbital fissure. ${ }^{3}$ In this article, we report a patient diagnosed with neuro-BD with diplopia, ipsilateral headache and ophthalmoplegia associated with cavernous sinus thrombosis, leading to Tolosa-Hunt-like syndrome.

\section{CASE REPORT}

A 53-year-old female patient visited our clinic with complaints of sudden onset of orbital pain, ipsilateral headache and intermittent diplopia. The severe, lancinating pain was aggravated with eyeball movements. Previously, she had been diagnosed with $\mathrm{BD}$ based on recurrent oral and

Received: December 12, 2018 Accepted: March 27, 2019 Published online: June 25, 2019

Correspondence: Changhoon Lee, MD. Department of Internal Medicine, Wonkwang University Hospital, 54538 Iksan, South Korea. Tel: 82638592662 e-mail:Ich110@nate.com 

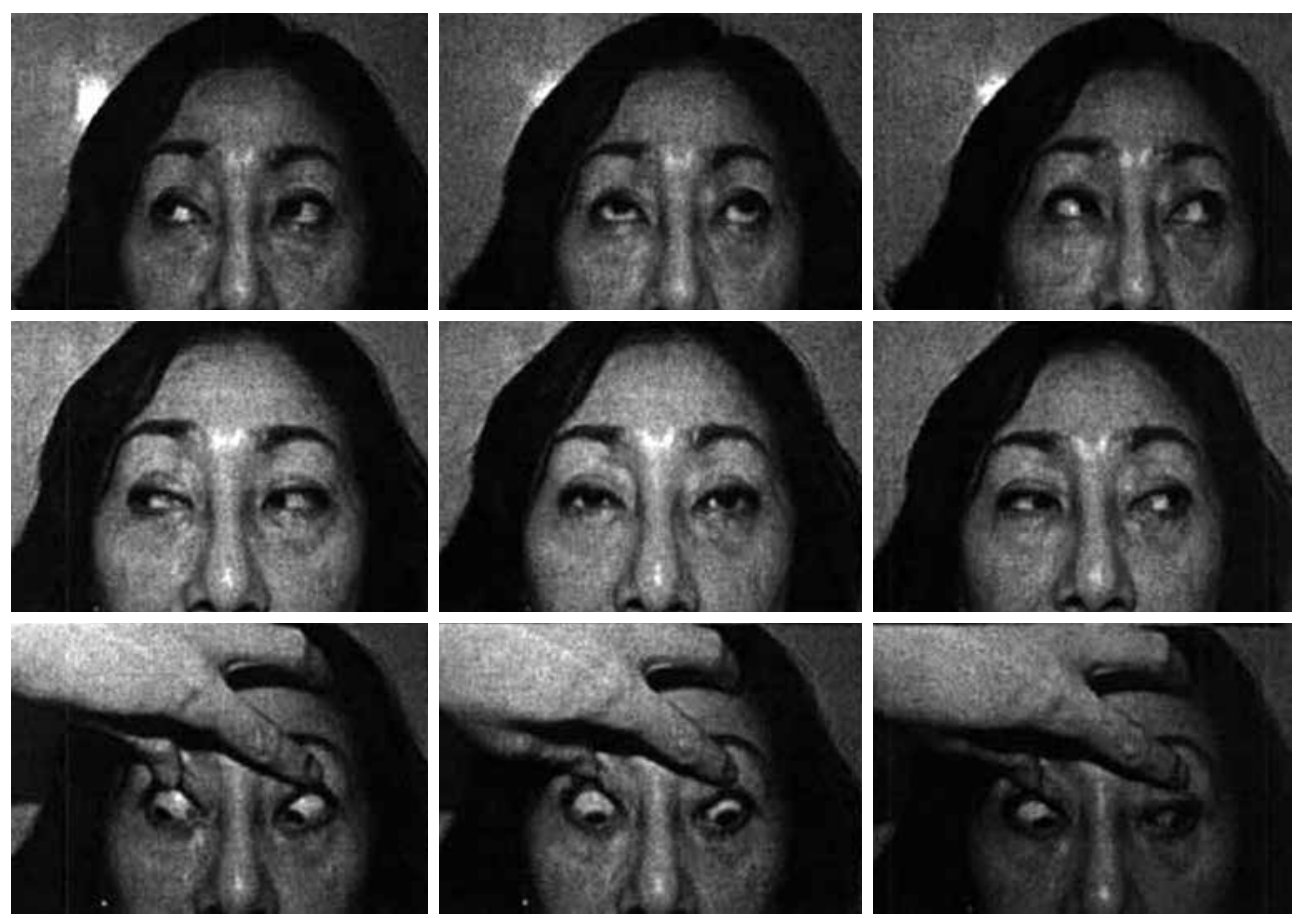

Figure 1. Images illustrating nine gazes showing left inferior rectus palsy and left-sided hypertropia.

This picture is posted with informed consent of patient for publication.

genital ulcers and a positive pathergy test. She tested positive for human leucocyte antigen B51. She remained on colchicine $1.2 \mathrm{mg} /$ day and had been asymptomatic for 12 years. She denied a history of hypertension, diabetes, malignancy, asthma or hyperthyroidism. The pupils reacted normally to light and were of equal size. Visual acuity was 20/20 in each eye. Mild hypertropia was observed on the left. Limited inferior rectus movement was found among the nine cardinal extraocular movements associated with diplopia (Figure 1). Neither papilledema nor raised intraocular pressure was found. The remaining neurological examination was normal. Laboratory parameters including complete blood count, muscle enzymes, sedimentation rate, C-reactive protein, antinuclear antibodies, double-stranded deoxyribonucleic acid antibodies, anti-neutrophil cytoplasmic antibodies, C3, C4 antiphospholipid, anticardiolipin, and $\beta 2$ glycoprotein antibodies, proteins $\mathrm{C}$ and $\mathrm{S}$, and thyroid function were all within normal range. Magnetic resonance imaging (MRI) of the brain demonstrated bulging and expansion of the left cavernous sinus (Figure 2) due to thrombosis. Cerebrospinal fluid examination was unrevealing. Finally, she was diagnosed with Tolosa-Hunt-like syndrome associated with $\mathrm{BD}$. The patient was started on a course of intravenous methylprednisolone $(1 \mathrm{~g} /$ day for five days). On day three of the treatment,

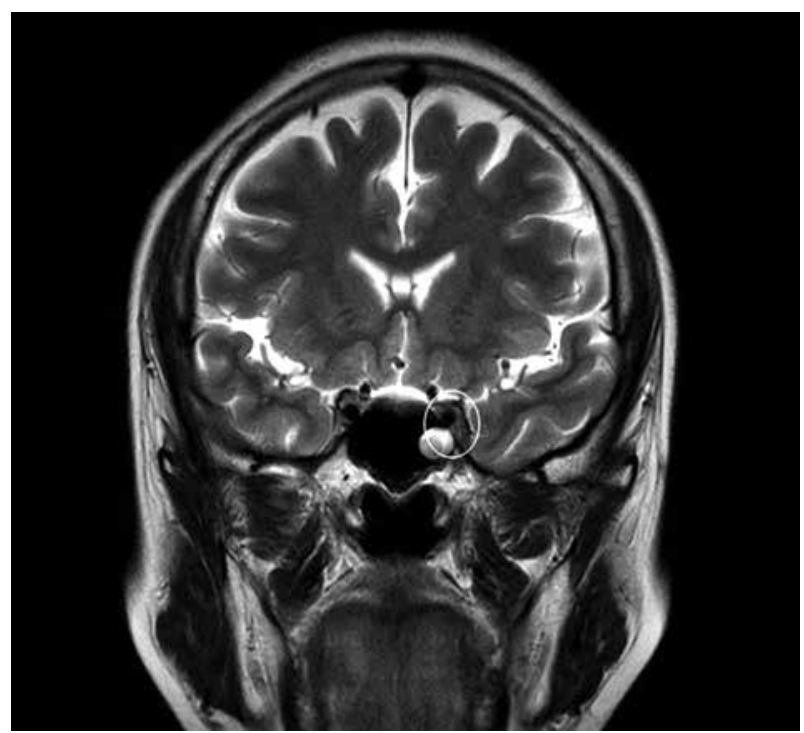

Figure 2. Brain magnetic resonance imaging with $\mathrm{T}_{2} \mathrm{~W}$ coronal image showing a lesion involving left cavernous sinus leading to asymmetrical lateral bulge and expansion (circle). 
she had a considerable relief of retro-orbital pain and headache. Two weeks later, noticeable improvement in ophthalmoplegia was observed following treatment with oral prednisolone $50 \mathrm{mg} /$ day. After eight weeks, she showed complete resolution of diplopia. Six months later, the patient remained on oral prednisolone $7.5 \mathrm{mg} /$ day without recurrence.

\section{DISCUSSION}

The pathophysiology of $\mathrm{BD}$ is characterized by inflammation of small arteries and veins, and thrombosis as a result of vasculitis of the vasa vasorum. ${ }^{4}$ Endothelial dysfunction caused by vascular inflammation is primarily responsible for the increased frequency of thrombosis. ${ }^{5}$

The prevalence of neurological manifestations in patients with $\mathrm{BD}$ varies between $5 \%$ and 30\%. ${ }^{6}$ Our case manifesting neuro-BD, presented with CVST. CVST occurs in $18 \%$ of neuroBD. ${ }^{2}$ The sites frequently involved include the superior sagittal sinus (64\%), the transverse sinus (61\%), the sigmoid sinus, and the straight sinus. However, cavernous sinus thrombosis occurs in only $2.9 \%$ of patients with CVST. ${ }^{2}$

Bouchez et al. ${ }^{7}$ first reported a 52-year-old female BD patient who spontaneously recovered from multiple cerebral venous thrombosis including cavernous sinus. She presented with retro-orbital pain, conjunctival swelling and hemorrhage, and hypoesthesia of the ophthalmic territory, without accompanying neurological symptoms such as ophthalmoplegia unlike our patient. The infiltration of the cavernous sinus may result in compressive neuropathy of the oculomotor, trochlear, and abducens cranial nerves inside the cavernous sinus. ${ }^{8}$ Our patient showed limitation of inferior rectus movement, and therefore, was suspected of oculomotor nerve involvement due to thrombosis as a complication associated with BD.

Generally, the prognosis of CVST is favorable. A majority (90\%) of the patients received corticosteroids and more than half were treated with other immunomodulatory drugs (azathioprine and cyclophosphamide). ${ }^{2}$ In addition to corticosteroids, anticoagulation therapy was used in most of the cases with CVST in BD. ${ }^{2}$ Saadoun et al. ${ }^{6}$ evaluated 64 patients with CVST out of 820 patients diagnosed with BD and
54 patients were treated with corticosteroids, and 32 with immunomodulators (cyclophosphamide and azathioprine). Anticoagulants were used to treat 62 of 64 patients manifesting acute CVST. Our patient was treated with intravenous highdose prednisolone for cavernous sinus thrombosis. Anticoagulation may not be necessary because of effective early response with corticosteroid treatment. We assumed that the patient presented with Tolosa-Hunt-like syndrome features such as painful ophthalmoplegia and diplopia.

Tolosa-Hunt syndrome is characterized by painful ophthalmoplegia with recurrent unilateral orbital pain and ipsilateral third, fourth and/or sixth cranial palsies, promptly resolved by corticosteroids. The annual incidence has been reported to be one case per million. ${ }^{9}$ The etiology of THS has been attributed to nonspecific granulomatous inflammation of uncertain etiology involving the septa and the wall of the cavernous sinus characterized by lymphocyte and plasma cell infiltration, formation of giant cell granulomas, and fibroblast proliferation. ${ }^{10}$ In general, THS is self-limiting, and promptly relieved with a short course of corticosteroids without longterm complications. Ophthalmoparesis usually requires a long course of corticosteroid therapy. ${ }^{11}$ Lasam et al. ${ }^{12}$ reported Tolosa-Hunt-like syndrome associated with progressive second, third, fifth, sixth, and eighth cranial nerve palsy in a diabetes mellitus patient. MRI of the brain showed cavernous sinus involvement.

In conclusion, we experienced the first case of cavernous sinus thrombosis as a complication of $\mathrm{BD}$ with very rare neurological symptoms mimicking THS such as ophthalmoplegia, ipsilateral headache and diplopia treated with corticosteroids. To our knowledge, the concomitant occurrence of Tolosa-Hunt-like syndrome and BD has not been reported until now.

\section{Declaration of conflicting interests}

The authors declared no conflicts of interest with respect to the authorship and/or publication of this article.

\section{Funding}

This study was supported by Wonkwang University grant in 2018. 


\section{REFERENCES}

1. Kir S, Akyol L, Özgen M, Pinar Günbey H, Sayarlioğlu M. Ptosis and Mass Like Lesions in Behçet's Disease: A Rare Presentation. Arch Rheumatol 2017;33:221-4.

2. de Sousa DA, Mestre T, Ferro J. Response to comment on "Cerebral venous thrombosis in Behcet's disease: a systematic review" by Afshin Borhani-Haghighi and Anahid Safari. J Neurol 2011;258:908-9.

3. Murtaza G, Konowitz N, Lu H, Faqah A, Kuruvilla A. An interesting case of tolosa-hunt syndrome in a young male. J Investig Med High Impact Case Rep 2017;5:2324709616689478.

4. Evereklioglu C, Er H, Türköz Y, Cekmen M. Serum levels of TNF-alpha, sIL-2R, IL-6, and IL-8 are increased and associated with elevated lipid peroxidation in patients with Behçet's disease. Mediators Inflamm 2002;11:87-93.

5. Evereklioglu C. Current concepts in the etiology and treatment of Behçet disease. Surv Ophthalmol 2005;50:297-350.

6. Saadoun D, Wechsler B, Resche-Rigon M, Trad S, Le Thi Huong D, Sbai A, et al. Cerebral venous thrombosis in Behçet's disease. Arthritis Rheum 2009;61:518-26.

7. Bouchez B, Arnott G, Caron JC, Guerouaou D, Clarisse G, Blondel $\mathrm{M}$, et al. Cerebral venous thrombosis with involvement of the cavernous sinus. Initial manifestation of Behçet's disease?. Rev Otoneuroophtalmol 1984;56:447-53.

8. Chaudhary V, Venu S, Deswal J. Pain ful ophthalmoplegia due to Tolosa-Hunt syn drome: a case re port. Iternational Journal of Medical Science and Public Health 2016;5:1045-8.

9. Iaconetta G, Stella L, Esposito M, Cappabianca P. Tolosa-Hunt syndrome extending in the cerebellopontine angle. Cephalalgia 2005;25:746-50.

10. Tolosa E. Periarteritic lesions of the carotid siphon with the clinical features of a carotid infraclinoidal aneurysm. J Neurol Neurosurg Psychiatry 1954;17:300-2.

11. Foubert-Samier A, Sibon I, Maire JP, Tison F. Longterm cure of Tolosa-Hunt syndrome after low-dose focal radiotherapy. Headache 2005;45:389-91.

12. Lasam G, Kapur S. A rare case of tolosa-hunt-like syndrome in a poorly controlled diabetes mellitus. Case Rep Med 2016;2016:9763621. 